\title{
Distribution of the Antigenic Substance among Absidia cylindrospora, Mucor hiemalis, and Rhizopus nigricans
}

\author{
Osamu Hayashi, Toshiro Yadomae and Toshio Miyazaki* \\ Department of Microbial Chemistry, Tokyo College of Pharmacy, \\ Hachioji, Tokyo 192-03, Japan
}

[Received for Publication: 9 January 1979]

\begin{abstract}
The slide-cultured mycelium of Absidia cylindrospora was stained brightly by either a direct or an indirect immunofluorescence staining method using FITCconjugated rabbit anti-ACE immunoglobulin. The paraffin-sections of Mucor hiemalis, and Rhizopus nigricans exhibited fluorescence as well as that of A. cylindrospora by the same methods. These facts indicated that immunological crossreactivity exist among these organisms. An uniformly distribution of the fluorescence was observed on the younger and older hyphae of $A$. cylindrospora, and the sporangiospores were also stained with the fluorescent conjugate.
\end{abstract}

A fluorescent antibody technique has offered a means for the study of the microscopic localization of antigenic substances on many mammalian cells and microorganisms. In the field of medical mycology, the fluorescent antibody technique has often been used for the detection and identification of pathogenic fungi ${ }^{1)}$.

In the previous papers ${ }^{2,3)}$, we suggested that some part of the serologically active substance from Absidia cylindrospora, might be loosely bound on the surface of the mycelium and easily released from the surface. We also showed the crossreactivity among some Mucorales by using the antiserum (anti-ACE serum) to $A$. cylindrospora. In the present paper, we examined the fluorescent antibody staining for the slide-cultured mycelium of $A$. cylindrospora and the paraffin-sections of $A$. cylindrospora, Mucor hiemalis, and Rhizopus nigricans by either a direct or an indirect method.

Furthermore, the fluorescent antibody staining was examined for the sporangiospore, germinanting hyphae, and the purified cell wall of $A$. cylindrospora by an indirect method.

\section{MATERIALS AND METHODS}

Organisms and culture methods. $A$. cylindrospora IFO 4000, $M$. hiemalis IFO 5305, and

*'To whom inquires should be addressed.
R. nigricans IAM 6070 were used in this study. Pythium debaryanum IFO 5919 was employed as a control. A. cylindrospora was cultured at $28^{\circ} \mathrm{C}$ by the following two methods; (a) slide culture on Sabouraud's glucose agar medium for 3 to 5 days and (b) shaking culture in Sabouraud's broth for 20 days. M. hiemalis and $R$. nigricans were cultured in Sabouraud's broth with shaking.

Spore suspension and purified cell wall of $A$. cylindrospora were prepared as described previously ${ }^{2}$.

To obtain germinating hyphae sporangiospores of $A$. cylindrospora were cultured in the liquid medium for $24 \mathrm{hrs}$ with agitation.

Mycelia and the germinating hyphae thus obtained were washed with cold saline and fixed with $10 \%(\mathrm{v} / \mathrm{v})$ formalin for $5 \mathrm{~min}$. at room temperature.

Preparations of the slides for fluorescent antibody staining. Spores, cell walls, and germinating hyphae of $A$. cylindrospora were suspended in distilled water and smeared on gelatincoated slide glasses. The used slide glasses were for fluorescent microscopy (Matsunami glass Ind. Ltd. Japan).

Slide-cultured mycelia of $A$. cylindrospora were stained with fluorescent antibody immediately 
after eliminating the agar medium and fixing with formalin.

The mycelia of $A$. cylindrospora, $M$. hiemalis, and $R$. nigricans, which were cultured in the liquid media (b), were embedded in paraffin after dehydration and then sectioned by a Jung type microtome (Yamato Koki Ltd., Japan). The sections $(6 \mu$ in thickness) were mounted on gelatin-coated slides which were moistened by wet brush and were dewaxed after air-drying.

Preparations of rabbit immunoglobulin and the fluorescent conjugates. Rabbit antiserum (anti-ACE serum) against the nondialyzable fraction of the culture filtrate of $A$. cylindrospora was prepared as described previously ${ }^{2)}$ and stored in a deepfreezer untill use.

The antiserum was diluted to two-fold with saline, and its globulin fraction was obtained by repeated precipitation with one-third saturated ammonium sulfate for 3 times. The precipitated globulin was centrifuged, redissolved in a small volume of saline, and dialyzed throughly against saline at $4^{\circ} \mathrm{C}$. The protein content of this solution was adjusted to $1 \%(\mathrm{w} / \mathrm{v})$ with saline. The globulin was labeled with fluorescein isothiocyanate (FITC) (ICN Pharmaceutical Inc., Cleveland) in $0.05 \mathrm{M}$ carbonate-bicarbonate buffer ( $\mathrm{pH} 9.5$ ) containing $0.1 \mathrm{M} \mathrm{NaCl}$ for $5 \mathrm{~h}$ at $4^{\circ} \mathrm{C}$. The labeled immunoglobulin fractions were passed through a column of Sephadex G-25 (fine) in order to eliminate excess FITC.

The fluorescent conjugates were further purified by a DEAE-cellulose DE-23 (Whatman Ltd., England) ion exchange column equilibrated with $0.005 \mathrm{M}$ sodium phosphate buffer ( $\mathrm{pH} 7.0$ ) containing $0.1 \mathrm{M} \mathrm{NaCl}$. The conjugation ratio of FITC to protein ( $\mathrm{F} / \mathrm{P}$ ratio) was estimated by measuring absorptions at $495 \mathrm{~nm}$ and $280 \mathrm{~nm}$ with spectrometer and then calculation by the formula.

The fractions showing the $\mathrm{F} / \mathrm{P}$ ratios of 0.8 to 1.7 were collected and concentrated to a small volume with dry powder of Sephadex G-200. The conjugates were dialyzed against $0.15 \mathrm{M}$ phosphate-buffered saline ( $\mathrm{pH} 7.2)$ and then stored at $4{ }^{\circ} \mathrm{C}$ in the presence of $0.1 \%(\mathrm{w} / \mathrm{v}) \mathrm{NaN}_{3}$.

Fluorescent antibody staining methods. (i) Direct method. Formalinized hyphal specimens were allowed to react directly with fluorescein-conjugated rabbit anti-ACE immunoglobulin for $1 \mathrm{hr}$ at $37^{\circ} \mathrm{C}$ followed by washing several times with phosphate-buffered saline. The slides were then rinsed in distilled water, air-dried, and the hyphal specimens on the slides were mounted with carbonate-buffered glycerin $(1: 9 \mathrm{v} / \mathrm{v})(\mathrm{pH} 9.5)$. Formalinized mycelia which had reacted with non-diluted anti-ACE serum before staining with FITC-conjugated rabbit antiACE immunoglobulin were employed as controls. $P$. debaryanum was also used as a control.

(ii) Indirect method. An indirect staining method was also examined by using the antiglobulin method of Weller \& Coons ${ }^{4)}$. A few drops of unlabeled rabbit anti-ACE serum were layered on the specimens and incubated for 60 min. at $37^{\circ} \mathrm{C}$. The slides were then washed several times with phosphate buffered saline, rinsed with water, and dried. FITC-conjugated goat anti-rabbit immunoglobulin (MILES-YEDA, Ltd., Israel) was applied to the specimens for 60 min. This fluorescent conjugate was used at a dilution of $1: 4$ to avoid non-specific staining effects. Washing and mounting were carried out as described above in the direct method.

Fluorescent microscopy and photomicrography. The treated preparations were examined under a Tiyoda fluorescent microscope FM-200A (Tiyoda Optical Co. Ltd., Japan) equipped with an Osram BHO-200 mercury vaporlamp. A Tiyoda UV exciter filter was used in combination with a Wratten 2B barrier filter. For photomicrography a Tiyoda $\mathrm{BV}$ exciter filter and a Y-51 barrier filter were employed. Photographas were made on Kodak High Speed Ektachrome Daylight ASA 200 using exposures of 60 to 120 sec.

\section{RESULTS}

Serological properties of FITG-conjugated rabbit anti-ACE immunoglobulin. The $F / P$ ratio of the finally concentrated conjugate was 1.15. Immunoelectrophoresis of the conjugate against goat anti-normal rabbit serum using $0.087 \mathrm{M}$ veronal buffer ( $\mathrm{pH} 8.6$ ) showed a single precipitin arc, which was identified with immunoglobulin G. In the immunodiffusion test, the conjugate showed a fused single precipitin line with either the crude ACE or the partially purified ACI. 


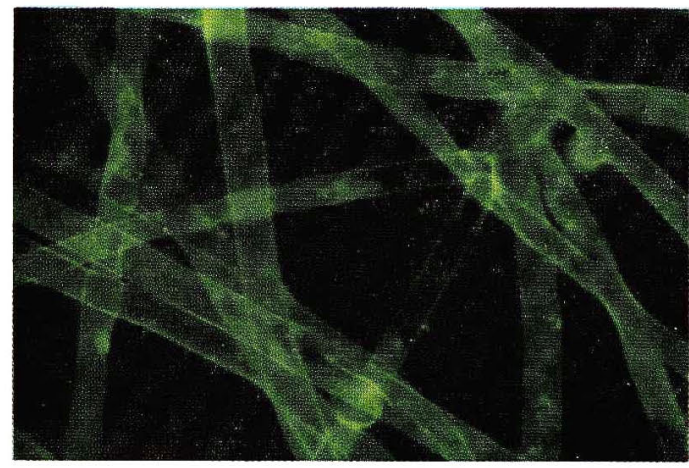

Fig. 1. Specific fluorescence of slide-cultured mycelium of Absidia cylindrospora stained by indirect method. $\times 400$

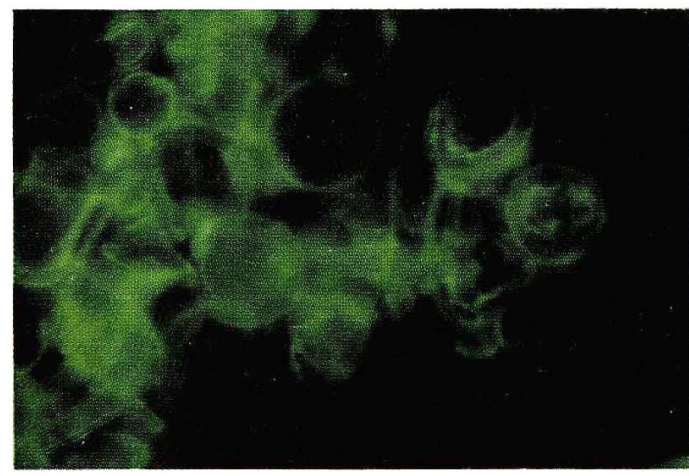

Fig. 3. Specific fluorescence of the paraffin-sections of Mucor hiemalis. $\times 400$

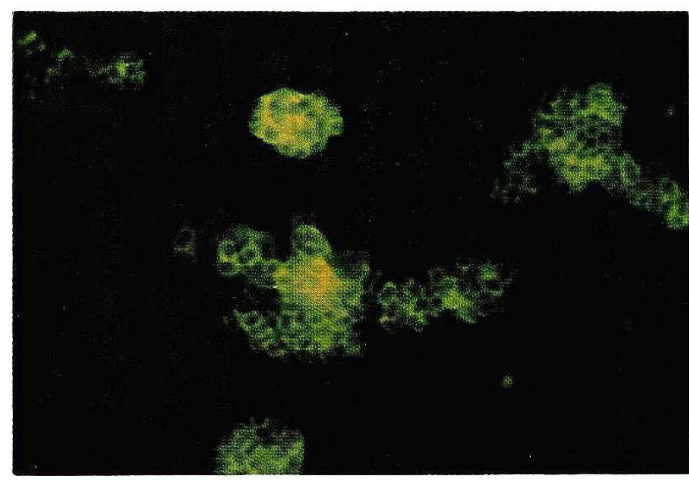

Fig. 8. Specific fluorescence of the sporangiospores of Absidia cylindrospora stained by indirect method. $\times 400$

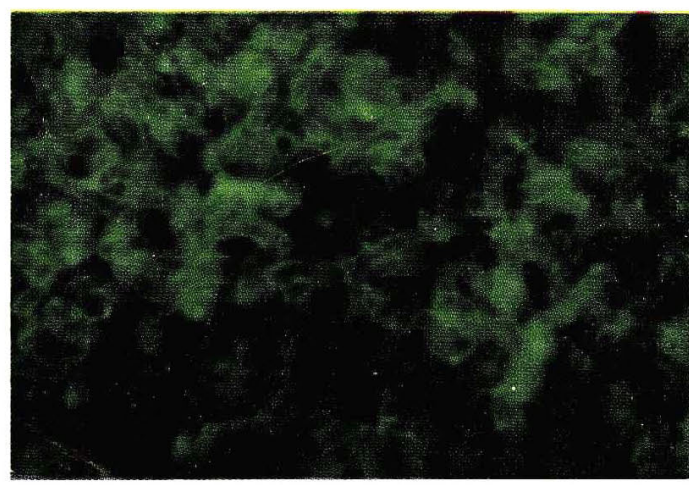

Fig. 2. Specific fluorescence of the paraffin-sections of Absidia cylindrospora. $\times 400$

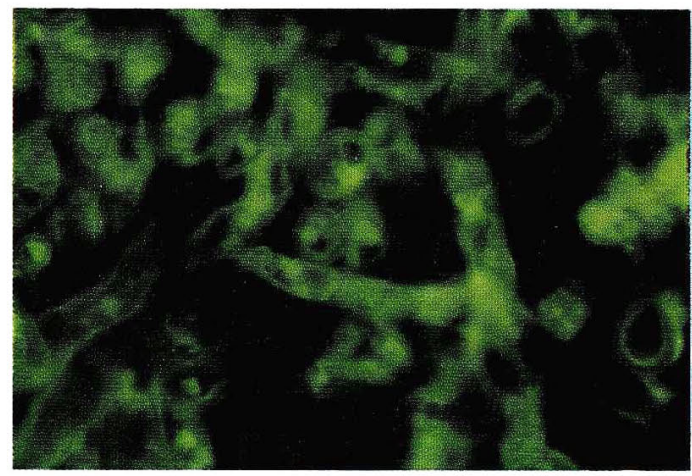

Fig. 4. Specific fluorescence of the paraffin-sections of Rhizopus nigricans. $\times 400$

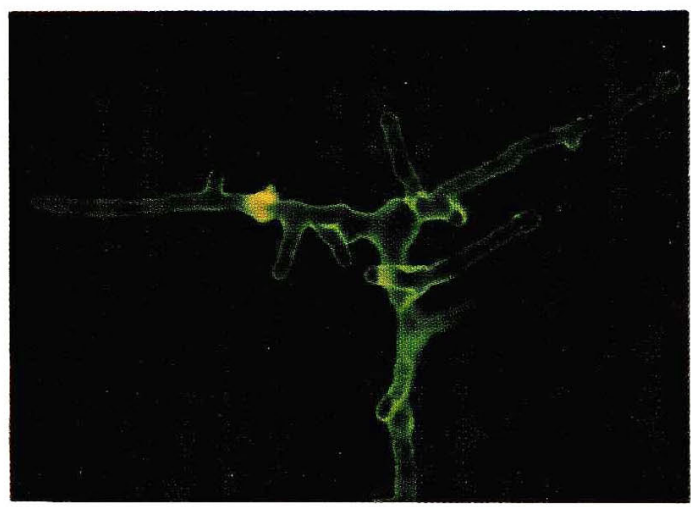

Fig. 9. Specific fluorescence of germinating hyphae of Absidia cylindrospora stained by indirect method. $\times 400$ 


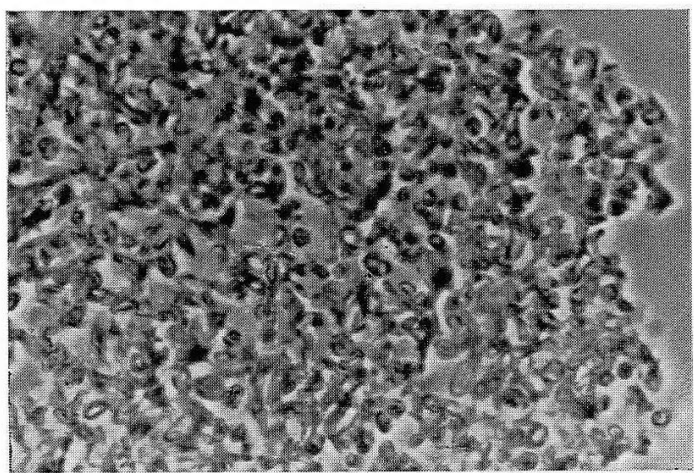

Fig. 5. Paraffin-sections of Absidia cylindrospora by phase contrast microscopy. $\times 400$

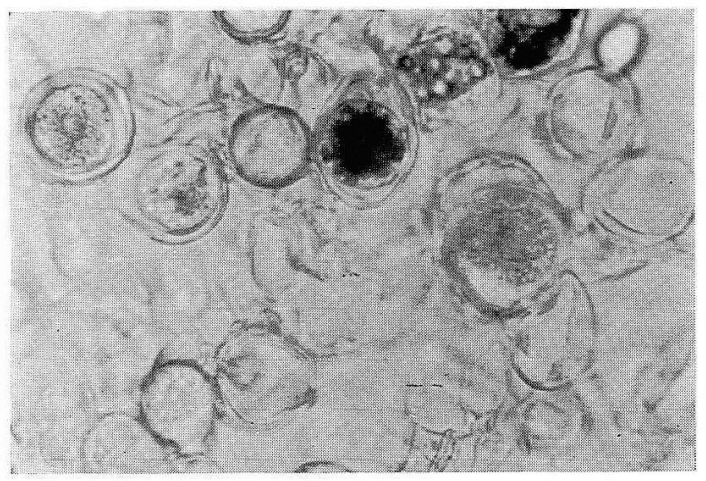

Fig. 6. Paraffin-sections of Mucor hiemalis by phase contrast microscopy. $\times 400$

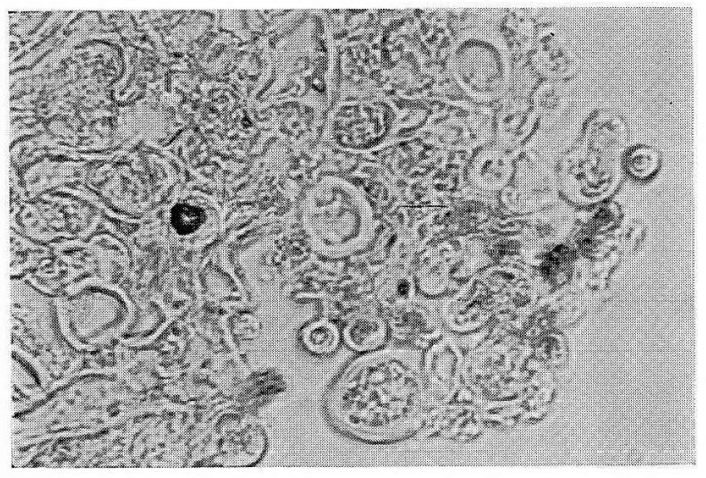

Fig. 7. Paraffin-section of Rhizopus nigricans by phase contrast micrescopy. $\times 400$

Fluorescent staining of $\boldsymbol{A}$. cylindrospora, $M$. hiemalis, and $R$. nigricans by a direct or an indirect method. As shown in Fig. 1, the slide-cultured mycelium of $A$. cylindrospora showed fluorescence at the hyphal wall surface by the direct and indirect methods but the intensity of the fluorescence which was observed in the hyphae stained by the direct method was much less than that stained by the indirect method (photo. not shown). Both the direct and indirect methods stained the paraffin-sections of the mycelia of $M$. hiemalis and $R$. nigricans as well as that of $A$. cylindrospora (Figs. 2, 3, and 4). Pythium debaryanum, used as a control, failed to be stained at any. As shown in Figs. 5, 6, and 7, transvers sections and a few longitudinal sections of the hyphae of these organisms were found in the paraffin-sections.

Fluorescent staining of the sporangiospore, germinating hyphae, and cell wall of A. cylindrospora by an indirect method. As shown in Figs. 8 and 9, fluorescence was observed in the spores and germinating hyphae. The fluorescence in the germinating hyphae distributed uniformly. The purified cell wall of $A$. cylindrospora showed faint fluorescence (no photo).

\section{DISGUSSION}

The serologically active substance was partially purified by concanavalin A-Sepharose affinity chromatography ${ }^{2}$ from ACI which was prepared from the supernatant of the disintegrated mycelium of $A$. cylindrospora. Similarly, partially purified serologically active substances, MhI-B and RnI-B, were obtained from the mycelia of $M$. hiemalis and $R$. nigricans, respectively. The cross-reactivity among $A$. cylindrospora, $M$. hiemalis, and $R$. nigricans has already been shown by the precipitin reaction of the ACI-B, MhI-B, and RnI-B against the anti-ACE serum ${ }^{3)}$. Moreover, we have reported that these active substances commonly contained mannoside units and that the precipitation of these were inhibited by the addition of linear $\alpha-(1 \rightarrow 6)$ linked mannooligosaccharides $^{5)}$. Uetsuka, Mine and Hotchi ${ }^{6)}$ reported the cross-reaction among three organisms of Absidia lichtheimii, Mucor globosus, and Rhizopus nigricans by means of the fluorescent antibody technique.

In the present study, we used this fluorescent antibody technique and showed the crossreactions among $A$. cylindrospora, $M$. hiemalis, and $R$. nigricans distinctively. In the case of the 
fluorescence antibody staining of the slide-cultured mycelium, the hyphal wall surface of $A$. cylindrospora was stained brightly (Fig. 1) and in the case of the staining of the paraffin-sections of these three organisms, the hyphal wall periphery or the cytoplasmic regions were stained brightly (Figs. 2, 3, and 4). On the other hand, the purified cell wall of $A$. cylindrospora exhibited faint fluorescence. These findings suggest that at least significant part of the serologically active substance of $A$. cylindrospora is loosely bound and easily released from the hyphal surface as expected in the previous paper ${ }^{2)}$.

The sporangiospores of $A$. cylindrospora were also stained by the fluorescent conjugate (Fig. 8). This result was in agreement with the fact that the spores of $A$. lichtheimii were strongly stained by the fluorescent antibody prepared from the anti-whole cell serum of this fungus ${ }^{7}$.

On the electron microscopic study, Hawker and $\mathrm{Abbot}^{8)}$ showed that newly formed cell walls during germination of the sporangiospores of $R$. nigricans and $R$. sexualis resembled the walls of the mature vegitative hyphae. Bartnicki-Garcia ${ }^{9)}$ reported that almost all fungal hyphae including Mucorales grew by apical growth and that cell wall synthesis occurred in hyphal apex.

In our observation, the germinating hyphae of $A$. cylindrospora also showed uniform fluorescence at the hyphal wall periphery (Fig. 9) as found in the mature vegitative hyphae of the slide-cultured mycelium, but the distribution of the fluorescence was not limited to the apex of the germinating hyphae of $A$. cylindrospora.

From these facts, it is estimated that at least significant part of the antigenic substance of $A$. cylindrospora may be uniformly distributed on the surfaces of the younger and older hyphae.

\section{ACKNOWLEDGMENT}

We express our gratitude to Prof. M. Kikuchi, Department of Hygiene, Juntendo University School of Medicine, for the use of microtome, to Kyokuto Pharmaceutical Inc. Co. Ltd., for the use of the fluorescent microscopy, and to Mr. Ohshima, for his technical assistance.

\section{REFERENGES}

1) Kaplan, W. and Kaufman, L.: The application of fluorescent antibody techniques to medical mycology - a review. Sabouraudia, 1: 137-144, 1961.

2) Hayashi, O., Yamada, H., Yadomae, T. and Miyazaki, T.: Purification and partial characterization of the serologically active substance from Absidia cylindrospora. J. Gen. Microbiol., 106: 289-295, 1978.

3) Hayashi, O., Yadomae, T., Yamada, H. and Miyazaki, T.: Cross-reactivity of antiserum to Absidia cylindrospora among some mucorales and other fungi. J. Gen. Microbiol., 108: 345-347, 1978.

4) Weller, J.H. and Coons, A.H.: Fluorescent antibody studies with agents of Varicella and Herpes zoster propagated in vitro. Proc. Soc. Exp. Biol. Med., 86: 789—794, 1954.

5) Miyazaki, T., Hayashi, O., Ohshima, Y. and Yadomae, T.: Studies on fungal polysaccharides. XXIII. On the immunological determinant of the serologically active substances from Absidia cylindrospora, Mucor hiemalis, and Rhizopus nigricans. J. Gen. Microbiol., 111: 417-422, 1979.

6) Uetsuka, A., Mine, Y. and Hotchi, M.: Detection of fungi by means of fluorescent antibody techniques. Clinic All-Round (Japanese), 15: 317-328, 1966.

7) Hotchi, M.: The application of fluorescent antibody technique to the identification of pathogenic fungi in tissue specimens. Med. J. Shinshu Univ., 12: 123—139, 1967.

8) Hawker, L.E. and Abbot, P.McV.: An electron microscope study of maturation and germination of sporangiospores of two species of Rhizopus. J. Gen. Microbiol., 32: 295-298, 1963.

9) Bartnicki-Garcia, S.: Fundamental aspects of hyphal morphogenesis. Symp. Soc. Gen. Microbiol., 23: 245-267, 1973. 